\title{
Virtual colonoscopy detected colorectal polyps in asymptomatic patients with average risk of colorectal neoplasia
}

Pickhardt PJ, Choi JR, Hwang I, et al. Computed tomographic virtual colonoscopy to screen for colorectal neoplasia in asymptomatic adults. N Engl J Med 2003;349:2191-200.

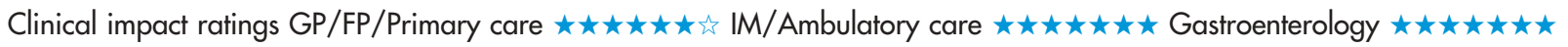

In asymptomatic patients with average risk for colorectal neoplasia, what is the accuracy of virtual colonoscopy for detecting colorectal polyps?

\section{METHODS}

ᄂ Design: blinded comparison of virtual colonoscopy (VC) with optical colonoscopy (OC)

(घ)

Setting: 3 medical centres in the US

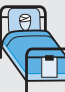

Patients: 1233 patients (mean age $58 \mathrm{y}, 59 \%$ men) with average risk of colorectal cancer. Exclusion criteria included positive result on guaiac based test of stool $\leqslant 6$ months before referral; iron deficiency anaemia in the previous 6 months; rectal bleeding or haematochezia or unintentional weight loss $>4.5 \mathrm{~kg}$ in the previous 12 months; $\mathrm{OC}$ in the previous 10 years; barium eneme in the previous 5 years; history of adenomatous polyps, colorectal cancer, or inflammatory bowel disease; and pregnancy.

Description of tests: VC was done before OC using a computed [D] U. II tomography (CT) protocol wherein pneumocolon was produced by insufflating room air through a rectal catheter immediately before scanning. A 4 or 8 channel CT scanner (GE LightSpeed or LightSpeed Ultra, General Electric Medical Systems) generated 2 and 3 dimensional ( $3 \mathrm{D}$ ) endoluminal displays of the colon and rectum while the patient held his or her breath in the supine and prone positions. The $3 \mathrm{D}$ display was used for the initial detection of polyps. OC used a standard commercial video colonoscope inserted to the caecum.

Diagnostic standard: after each segment was inspected, results of $\mathrm{VC}$ for that segment were revealed. If a polyp $\geqslant 5 \mathrm{~mm}$ in diameter was seen on $\mathrm{VC}$ but not on $\mathrm{OC}$, the endoscopist reexamined the segment to create the diagnostic standard (enhanced $O C$ ), and to capture false negative results on $O C$ that would otherwise be recorded as false positive results on VC.

12 Outcomes: sensitivity, specificity, and likelihood ratios.

\section{MAIN RESULTS}

554 adenomatous polyps were detected. The prevalence of polyps of diameters $\geqslant 6 \mathrm{~mm}, \geqslant 8 \mathrm{~mm}$, or $\geqslant 10 \mathrm{~mm}$, was $13.6 \%, 6.7 \%$, and $3.9 \%$, respectively. The diagnostic performance of VC at increasing polyp sizes is shown in the table Sensitivity for initial OC was slightly less than that of $\mathrm{VC}$ at polyp sizes $\geqslant 8 \mathrm{~mm}$. Of 55 polyps $(\geqslant 5 \mathrm{~mm}$ in

For correspondence: Dr P J Pickhardt, University School, Madison, WI, USA. ppickhard+@mail.radiology.wisc.edu Source of funding: Department of Defense. diameter) detected by VC but missed by initial OC, 21 (38\%) were adenomatous and measured $\geqslant 6 \mathrm{~mm}$ in diameter. OC was not as sensitive as VC for detecting advanced neoplasms (measuring $\geqslant 10$ $\mathrm{mm}$ ) (sensitivity according to the polyp $88.1 \% \vee 91.5 \%$ ). Of the 2 adenocarcinomas identified, VC detected both and initial OC missed 1 (an $11 \mathrm{~mm}$ polyp).

\section{CONCLUSION}

In asymptomatic patients with average risk of colorectal neoplasia, virtual colonoscopy was sensitive and specific for detecting colorectal polyps.

Abstract and commentary also appear in ACP Journal Club.

\section{Commentary-continued from previous page}

A II currently accepted tests for colorectal cancer screening-faecal occult blood tests, sigmoidoscopy, double contrast barium enema, and colonoscopy - are effective, but none is ideal. There is always room for another test with a different combination of such characteristics as accuracy, safety, convenience, comfort, cost, and availability. VC has been a promising option, 'but no rigorous evaluations of polyp detection have been done in people at average risk of colorectal neoplasia.

Now there is good information on how well VC detects clinically important polyps in average risk people. 2 strong studies, published within 4 months of each other, come to different conclusions. The study by Pickhardt et al says "CT virtual colonoscopy ... is an accurate screening method ... and compares favorably with optical colonoscopy..." The study by Cotton et al says "computed tomographic colonoscopy ... is not ready for widespread clinical application." I believe both are right. They ask different questions and get different answers.

Pickhardt et al ask whether state of the art VC under ideal circumstances can detect polyps in average risk people as well as conventional colonoscopy, the current gold standard. The test they studied had technological features, such as "electronic cleansing" (computer based removal of residual fluid), that are not generally available. Interpretation relied primarily on a 3 dimensional, rather than a 2 dimensional, approach to the detection of polyps, which is not generally used. Also, the 6 radiologists were specially trained, having done $\geqslant 25$ (and in some cases $>100$ ) such studies. Cotton et al on the other hand, studied the performance of VC under more ordinary circumstances, the kinds of settings where most patients would have the procedure.

Robert H Fletcher, MD, MSc Harvard Medical School

Boston, Massachusetts, USA

1 Fenlon HM, Nunes DP, Schroy PC 3rd, et al. A comparison of virtual and conventional colonoscopy for the detection of colorectal polyps. N Engl J Med 1999;341:1496-503.

Test characteristics of virtual colonoscopy for detecting colorectal adenomas *

\begin{tabular}{lllll}
\hline Polyp size & Sensitivity (95\% Cl) & Specificity (CI) & +LR & - LR \\
\hline$\geqslant 6 \mathrm{~mm}$ & $89 \%(83$ to 93$)$ & $80 \%(77$ to 82$)$ & 4.35 & 0.14 \\
$\geqslant 8 \mathrm{~mm}$ & $94 \%(86$ to 98$)$ & $92 \%(91$ to 94$)$ & 12.04 & 0.07 \\
$\geqslant 10 \mathrm{~mm}$ & $94 \%(83$ to 99$)$ & $96 \%(95$ to 97$)$ & 23.45 & 0.06 \\
\hline
\end{tabular}

*Diagnostic terms defined in glossary; LRs calculated from data in article 